\title{
Circuito de arranque suave para un motor monofásico de corriente alterna conectado a un Sistema Fotovoltaico
}

\section{Soft start circuit for a single phase alternating current motor connected to a Photovoltaic System}

BRAVO-TAPIA, Jeremías $\dagger^{*}$, GARCÍA-MARTÍNEZ, Eric, RAMOS-GUZMÁN, Francisco y ESTÉVEZ-MARTÍNEZ, Yoxkin

Instituto Tecnológico Superior de Acatlán de Osorio

ID $1^{\mathrm{er}}$ Autor: Jeremías, Bravo-Tapia / ORC ID: 0000-0001-9022-9076, CVU CONACYT ID: 996738

ID $1^{\text {er }}$ Coautor: Eric, García-Martínez / ORC ID: 0000-0002-0733-1916, CVU CONACYT ID: 1000483

ID $2^{\text {do }}$ Coautor: Francisco, Ramos-Guzmán / ORC ID: 0000-0003-0939-4707, CVU CONACYT ID: 680766

ID $3^{\text {er }}$ Coautor: Yoxkin, Estévez-Martínez / ORC ID: 0000-0003-3756-0573, CVU CONACYT ID: 224129

DOI: $10.35429 /$ JSI.2019.11.3.27.39

Recibido 16 de Junio, 2019; Aceptado 30 Septiembre, 2019

Resumen

Este trabajo describe el desarrollo de un circuito eléctrico para ser utilizado como Arrancador Suave en motores monofásicos de corriente alterna conectados a sistemas fotovoltaicos. El arranque directo de un motor conectado a un inversor activa su protección para minimizar y eliminar los altos picos de corriente que se generan debido a que no es capaz de soportar la corriente que el motor demanda; para contrarrestar esta situación, existen arrancadores suaves o variadores de frecuencia y otras técnicas utilizadas para motores trifásicos; sin embargo, no se ha hecho lo mismo para los motores monofásicos, por lo que el objetivo de esta investigación fué diseñar e implementar un circuito eléctrico de arranque suave para contrarrestar la marcha inicial de un motor monofásico de corriente alterna conectado a un sistema fotovoltaico. La metodología de desarrollo consistió en el diseño, la construcción, la implementación y la validación del circuito propuesto, utilizando para ello, un motor monofásico de $1 / 4 \mathrm{Hp}$ y un inversor de $1,500 \mathrm{~W}$. Los resultados obtenidos mostraron una reducción de la potencia con la cual inició el arranque directo que se observó de 930 watts, a 180 watts que presentó el motor con el circuito de arranque suave.

Arrancador, Suave, Monofásico

\begin{abstract}
This work presents the study of a circuit used as a Soft Starter for a single-phase AC motor connected to a photovoltaic system. The direct start of a motor connected to an inverter activates its protection because it is not able to withstand the current that the motor demands in its start, that is why a technique is proposed that minimizes and eliminates the high peaks of current that generate Currently there are soft starters or frequency variations and other soft start techniques that are used for three-phase motors, what is required for this case is a soft starter for single phase motors. The circuit used as a test for Soft Starter allows performing the phase control by varying the firing angle. The photovoltaic system with which the soft starter tests were carried out has an inverter of $1,500 \mathrm{~W}$ to which a single-phase motor of a quarter of $\mathrm{Hp}$ was connected. Measurements were made with a digital multimeter, a hook Wattmeter, a digital oscilloscope and a washing machine motor. The main bases for the development of Soft Starters are left connected to a photovoltaic inverter for the start of single-phase motors.
\end{abstract}

Starter, Soft, Mono-phase

Citación: BRAVO-TAPIA, Jeremías, GARCÍA-MARTÍNEZ, Eric, RAMOS-GUZMÁN, Francisco y ESTÉVEZMARTÍNEZ, Yoxkin. Circuito de arranque suave para un motor monofásico de corriente alterna conectado a un Sistema Fotovoltaico. Revista de Innovación Sistemática. 2019. 3-11:27-39

\footnotetext{
*Correspondencia al Autor (Correo electrónico: jrmsbt@ @otmail.com)

$\dagger$ Investigador contribuyendo como primer Autor.
} 


\section{Introducción}

Hoy en día al conectar un motor monofásico al inversor de un sistema fotovoltaico, la demanda de corriente que provoca durante el arranque es más elevada que la que proporciona el inversor, esto ocasiona que la protección del inversor se dispare constantemente ya que no es capaz de soporta la energía que demanda el motor durante el arranque, esto genera pérdidas en el consumo de energía porque descarga rápidamente el banco de baterías que puede provocar que el inversor se sobrecaliente o queme, dicho inversor tiene un costo elevado dependiendo de la marca y sus funciones; debido a la situación económica que se presenta en las zonas rurales y urbanas es difícil de conseguirlo y sería una inversión no necesaria para las demás funciones del sistema fotovoltaico, además la inversión en reparaciones no es adecuada porque en muchas de las ocasiones los componentes no se encuentran o terminan dañando más el circuito del inversor, lo cual generaría una nueva adquisición de un nuevo inversor de alta potencia.

Actualmente existen arrancadores suaves o variadores de frecuencia y otras técnicas de arranque suave que se utilizan para motores trifásicos y lo que se requiere para este caso es un arrancador suave para motores monofásicos.

En el Instituto Tecnológico Superior de Acatlán de Osorio (ITSAO), se busca mejorar el aprovechamiento de sistemas fotovoltaicos reduciendo su costo, con este proyecto se logra esa parte de reducción de costo y la mejora del aprovechamiento de los paneles solares, además beneficiará a todos los sectores que hacen uso y requieren hacer la implementación de sistemas fotovoltaicos a menor costo, tanto en el campo, en el hogar, en pequeñas y medianas empresas y a nivel industrial.

El objetivo principal es diseñar un circuito de arranque suave para contrarrestar la marcha inicial de un motor monofásico de corriente alterna conectado a un sistema fotovoltaico mediante dispositivos electrónicos.

La implementación de un Arrancador Suave conectado al inversor de un sistema fotovoltaico, permitirá reducir la alta demanda de corriente que un motor monofásico genera al arranque.

\section{Arrancadores suaves en motores monofásicos}

$\mathrm{El}$ arranque de motores convencional directo o estrella-triángulo origina problemas eléctricos, por lo que desde hace año los fabricantes vienen desarrollando soluciones para mitigar los efectos negativos que provocan estos picos de intensidad. La conexión de los motores de CA, utilizados para accionar ventiladores, trituradoras, agitadores, bombas, cintas transportadoras, etc., y cualquier equipo que realiza un trabajo duro en la industria, produce cotidianamente innecesarios picos de intensidad en las plantas de producción de todo el mundo. Estos arranques violentos causan daños de diferente naturaleza («TODOPRODUCTIVIDAD», 2011). Entre ellos los siguientes:

\section{a. Problemas eléctricos \\ b. Problemas mecánicos \\ c. Problemas funcionales}

Los arrancadores suaves son la solución idónea y sencilla para todos estos problemas. Con los arrancadores suaves es posible realizar arranques y paros suavemente, reduciendo al mínimo los esfuerzos eléctricos y mecánicos. Vamos a ver las características esenciales de estos equipos usando lo descrito por uno de los fabricantes típicos, ABB.

Un motor monofásico al no poseer par de arranque no puede iniciar marcha por sí solo. Es por esto que se utilizan para su arranque distintas técnicas eléctricas: devanados de fase partida, arranque por condensador y polos de estator sombreado (Orellana Zea \& Pintado Garate, 2019).

En el arranque de los motores que accionan las bombas fotovoltaicas se producen intensidades muy altas, así como un elevado par de arranque, que puede ser perjudicial para el motor desde el punto de vista mecánico. Para evitar estos inconvenientes se suele limitar la intensidad de arranque mediante el uso de autotransformadores, el arranque estrellatriángulo o la eliminación de resistencia en rotor y estator. Los arrancadores estáticos presentan ventajas frente a los métodos clásicos de arranque. Una de las ventajas de los arrancadores estáticos es que se obtiene un arranque suave que minimiza los efectos producidos por los golpes y las vibraciones. 
Mejoran el rendimiento de los motores y son muy útiles en electrobombas al permitir controlar el golpe de ariete (Miguel Llopis Morales, 2018).

El motor de inducción de jaula de ardilla monofásico (SPIM) se usa ampliamente en aplicaciones domésticas e industriales debido a su estructura áspera y simple. Diferentes tipos de aplicaciones traen diferentes requisitos óptimos de funcionamiento. El arranque del motor monofásico con la conexión de línea directa provoca una corriente de arranque alta y una ondulación de par alto en muchas aplicaciones. Debido a estos inconvenientes, el control de arranque del motor con los circuitos electrónicos de potencia es necesario donde se requiere el arranque suave (Ayyildiz, Shok, Soygenc, \& Ergene, 2018), ellos realizaron el desarrollo del diseño e implementación de un circuito de arranque suave para un motor monofásico y se presentan las salidas principales del circuito de control.

El motor de inducción monofásico (SPIM) tiene una alta corriente de arranque y necesita un interruptor centrífugo para desconectar el devanado auxiliar. El uso de arrancadores suaves o controladores de velocidad variable monofásicos puede resolver adecuadamente estos dos problemas. Sin embargo, estas soluciones son caras (Neri \& Lyra, 2006), ellos analizaron el nuevo suministro de topología para SPIM utilizando un TRIAC, llamado método de suministro de doble atenuador, que se centra en el control del ángulo entre los voltajes de bobinado principal $y$ auxiliar.

Un motor de inducción fotovoltaico está acoplado mecánicamente a la bomba de agua. Los paneles solares que esencialmente proporcionan voltaje de $\mathrm{CC}$ se utilizan para bombear agua utilizando un motor de inducción. Debido a su confiabilidad, confiabilidad, bajo valor y bajo costo de mantenimiento; el motor de inducción se utiliza como parte de la mayoría de las aplicaciones comerciales, industriales y de otro tipo. La salida requiere un control de velocidad variable ya que el motor requiere un arranque suave y el sol cambia constantemente de posición, por lo que la potencia generada por las células fotovoltaicas estacionarias varía en consecuencia.
Debido a la diferente situación de voltaje, el motor no produce el par máximo en una frecuencia de suministro específica. Por lo tanto, la frecuencia suministrada debe cambiarse para alcanzar la máxima potencia (Khan, Khan, \& Hussain, 2015). Se presenta un novedoso sistema de bombeo de agua con energía solar, especialmente adecuado para su uso en áreas rurales o remotas(Bhagyashree \& Khule, 2017) . En este esquema, el inversor acciona el motor de inducción, que acciona la bomba de agua. Además, la capacidad de arranque de un motor de inducción se vuelve bastante pobre debido a la caída en la tensión del sistema cuando arranca el motor. Por esta razón, la eficiencia general de los sistemas de accionamiento basados en un motor de inducción suministrados por un conjunto fotovoltaico es menor.

Para obtener la potencia de salida máxima del panel solar, el inversor funciona con un arranque suave para minimizar la corriente de arranque del motor utilizando FCMA tecnología. El uso de FCMA ayuda en los controles de velocidad variable, aumentando la vida útil de los componentes y reduciendo el costo de capital y el mantenimiento. También ayuda a mejorar la eficiencia del motor.

Existen sistemas de bombeo fotovoltaico donde el controlador incluye un arrancador suave para el motor de la bomba de agua para protección de los contactos de los relés de la bomba, los problemas de arranque directo no solo dañan los relés, también daña los circuitos electrónicos por la radiofrecuencia generada durante el arranque del motor. Existen bombas de corriente directa y de corriente alterna, se han utilizado circuitos de control de fase escalonada con TRIAC's («Adding a Soft Start to Water Pump Motors - Reducing Relay Burning Problems | Homemade Circuit Projects», s. f.).

A una temperatura y nivel de irradiancia, un generador FV tiene una característica I-V. Para maximizar la transferencia de energía, la carga eléctrica debe ser tal que su propia característica I-V intercepte a la del generador FV tan cerca del punto de máxima potencia como sea posible. El papel del circuito de acondicionamiento de potencia es proporcionar al motor/bomba la combinación más adecuada tensión/corriente, a la vez que asegurar que el generador FV opera en su punto de máxima potencia. 
Los convertidores DC/DC, debido a que su propósito principal es mantener tanto al generador como a la carga en su respectivo punto de trabajo óptimo, suelen ser seguidores del punto de máxima potencia (MPPT) y efectúan un ajuste de potencia para incrementar el rendimiento del sistema de bombeo a largo del día. Los inversores DC/AC tienen la principal misión de transformar la corriente continua de salida del generador en corriente alterna, apta para su utilización por motores AC (Miguel Alonso \& Faustino Chenlo, s. f.) y (González Ponce Abraham, 2016).

Los inversores de uso en bombeo fotovoltaico generan una salida monofásica o trifásica con voltaje y frecuencia variables. La variación de la frecuencia de salida permite a los motores operar a velocidades distintas de la velocidad nominal correspondiente a la frecuencia nominal de $50 / 60 \mathrm{~Hz}$ y así disminuir el umbral de irradiancia solar para el arranque de la bomba.

Carlo Gavazzi presenta el arrancador suave compacto y simple para motores de inducción de CA monofásicos de bajo par de arranque. Solo requiere dos ajustes sencillos para el par inicial y el ajuste del tiempo de la rampa de arranque («Arrancador suave de estado sólido monofásico Carlo Gavazzi RGTS Informática industrial - Arrancador suave de estado sólido monofásico», s. f.).

EasyStart TM: el arrancador suave más avanzado y efectivo para todas las aplicaciones de A / C. EasyStart TM es un arrancador suave único para su desarrollo personalizado diseñado para motores monofásicos. Emplea una secuencia de rampa de inicio de 4 partes que se optimiza automáticamente, lo que resulta en la corriente de arranque más baja posible. EasyStart puede ofrecer una reducción de la corriente de arranque del $65-75 \%$ en comparación con el LRA (amperaje de rotor bloqueado) de un compresor. EasyStart es la solución perfecta que permite que un acondicionador de aire o un compresor de refrigeración funcione con un generador, un inversor o una potencia limitada de la red pública cuando de otro modo no habría funcionado. También se puede aplicar a motores de compresores de aire y bombas de fluido («EasyStart 364 (3-ton) Single Phase Soft Starter for Air Conditioners - Micro-Air, Inc.», s. f.).

\section{Descripción del método}

La prueba a realizar con el desarrollo del circuito es observar el arranque progresivo para un motor monofásico de pequeña potencia para ser conectado al inversor de un sistema fotovoltaico autónomo, los casos a observar se muestran en las figuras 1, 2 y 3 en las que se logra visualizar la diferencia básica entre el arranque en línea directo (DoL), el arranque en estrella-triángulo y el arranque suave, en cuanto a tensión del motor (V), intensidad del motor (I) y par del motor (M) en relación con el tiempo (t) y velocidad del motor (n) (Kling, 2002).

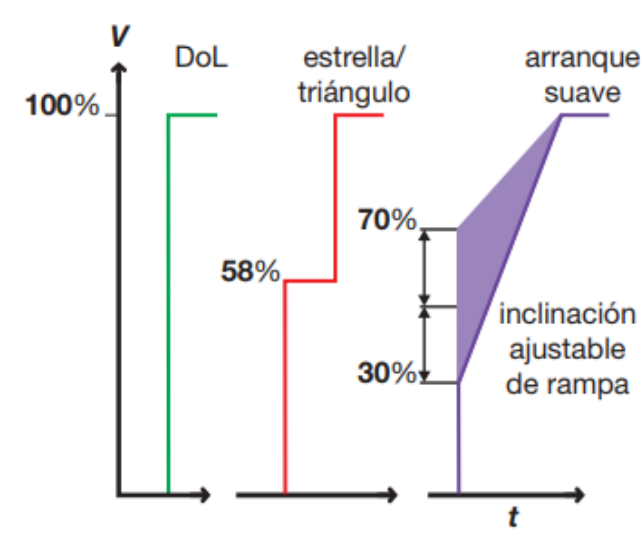

Figura 1 Curvas de tensión (Kling, 2002)

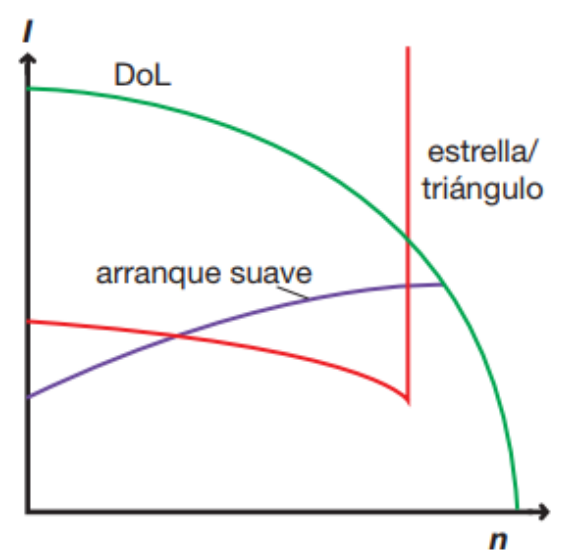

Figura 2 Curvas de corriente (Kling, 2002)

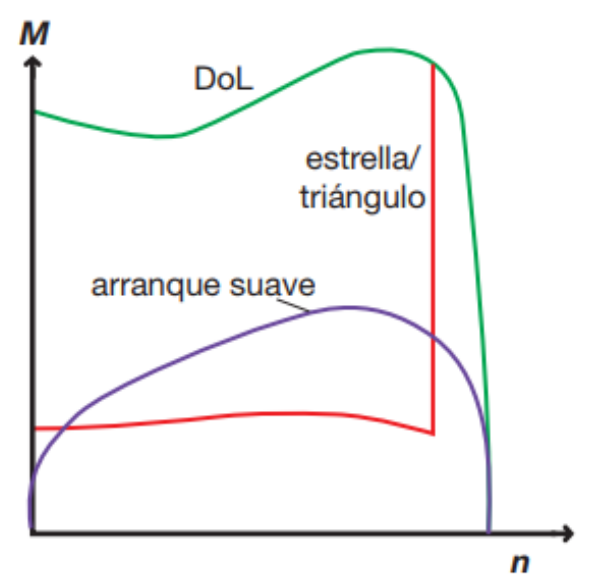

Figura 3 Curvas de par (Kling, 2002) 
El sistema se implementará utilizando semiconductores de potencia, cuyo gobierno se efectúa mediante circuitos integrados (CI) que realizan el control por ángulo de fase. El arranque directo es cuando toda la corriente se manda de forma unilateral, el arrancador mediante estrella-triangulo es cuando la tensión enviada es proporcional durante un cierto periodo de tiempo y el resto de la tensión se manda después de otro periodo, para el arrancador suave en la figura 3 se observa que se caracteriza por enviar una cierta tensión al principio de la señal y después de un determinado periodo de tiempo se utiliza una pendiente para que ésta vaya ajustando los niveles de voltaje que se van a ir enviando.

La intensidad del motor con el arranque directo empieza completamente a elevarse y según se vaya variando el periodo de tiempo se va bajando, con el arrancador de estrellatriangulo la corriente inicial no es tan elevada y tiene un pico de estabilización, mientras que con el arrancador suave la corriente empieza de manera baja y gradualmente se va estabilizando hasta alcanzar el valor nominal.

De igual manera para el par se tiene que en el arranque directo presenta una forma senoidal que una vez que se estabiliza el par cae completamente, en el estrella-triangulo el par se mantiene constante durante el arranque pero para estabilizarse sucede un proceso igual al de la corriente como se eleva el voltaje, también se eleva el par; mientras que con un arrancador suave sucede un proceso más o menos parecido al que se tiene para la corriente empieza de una manera muy baja y sube gradualmente presenta un pico relativamente pequeño y baja pero se vuelve constante.

A continuación, en la figura 4 se muestra el diagrama correspondiente a la ubicación de cómo estará conectado el Arrancador Suave al sistema fotovoltaico. Queda conectado entre el inversor y la carga o el motor.

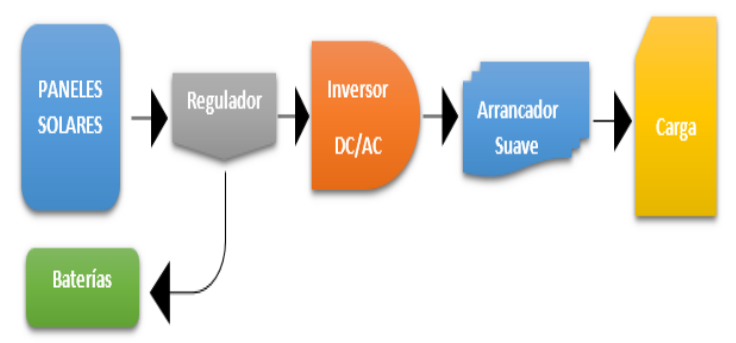

Figura 4 Estructura de conexión del Arrancador Suave
Al visualizar y comparar las diferentes técnicas de arranque suave de motores monofásicos se identificó que la forma más adecuada para este caso es utilizando TRIAC's a través del corte de fase escalonado, para iniciar el arranque suave lento y el final lento o el circuito de parada lenta de modo que los motores puedan pasar por una acción de parada de arranque gradual en lugar de encender/apagar abruptamente. Los tiristores son ampliamente utilizados para controlar la corriente alterna, ya que cuando se activan correctamente su pérdida de potencia es mínima debido a la trayectoria de baja resistencia entre ánodo y cátodo, también los componentes externos son mínimos y menos complejos, lo que lo convierten en el dispositivo perfecto (Castro, Rios, \& Merino, 2017).

Se hace uso de la electrónica de Potencia que tiene efectos sobre el control de la energía eléctrica; con el objeto de adaptar de manera eficiente el uso de la electricidad, para el caso de los motores eléctricos de corriente alterna AC (Ludeña, Milton, Lapo \& Douglas, 2018), utilizando como principal componente el triodo de corriente alterna TRIAC.

En la figura 5 se muestra el diagrama electrónico fundamental para el control de potencia en cargas de corriente alterna; la ventaja de esta configuración es que durante la parte negativa de la señal de entrada se obtendrá el mismo tipo de respuesta ya que tanto el DIAC como el TRIAC se pueden encender en la dirección inversa. También se muestra la forma de onda resultante de la corriente a través de la carga; si se modifica el resistor $\mathrm{R}$, se puede controlar el ángulo de conducción. Existen unidades disponibles capaces de manejar cargas de más de $10 \mathrm{~kW}$ (Boylestad \& Nashelsky, 2009).

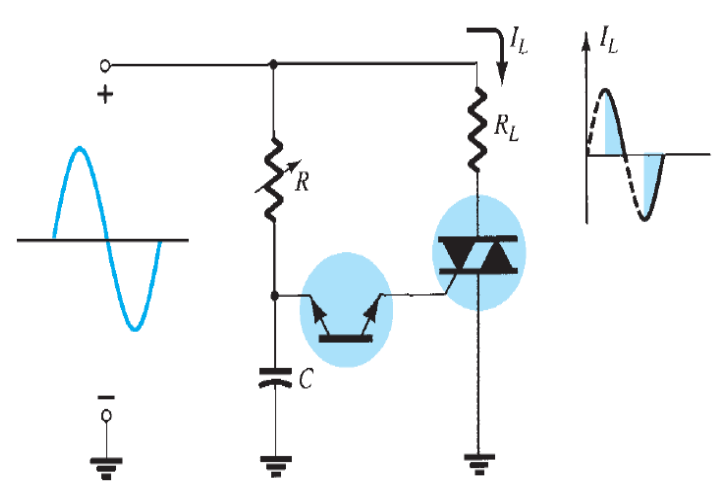

Figura 5 Control de potencia con tiristores con TRIAC y DIAC (Boylestad \& Nashelsky, 2009) y (Maloney, 2006) 
El circuito de disparo de TRIAC mostrado en la figura 6 está mejorado para establecer un ajuste más amplio del rango del ángulo de retardo de disparo del TRIAC debido a que una sola red RC no puede retardar mucho el disparo. El ritmo de carga del capacitor $\mathrm{C}$ se establece por medio de la resistencia $\mathrm{R}_{2}$. Para una $\mathrm{R}_{2}$ grande, la velocidad de carga es lenta, lo que produce un retardo de disparo largo y una corriente de carga promedio pequeña. Para una $\mathrm{R}_{2}$ pequeña, la velocidad de carga es rápida, el ángulo de retardo de disparo es pequeño y la corriente de carga es alta (Maloney, 2006).

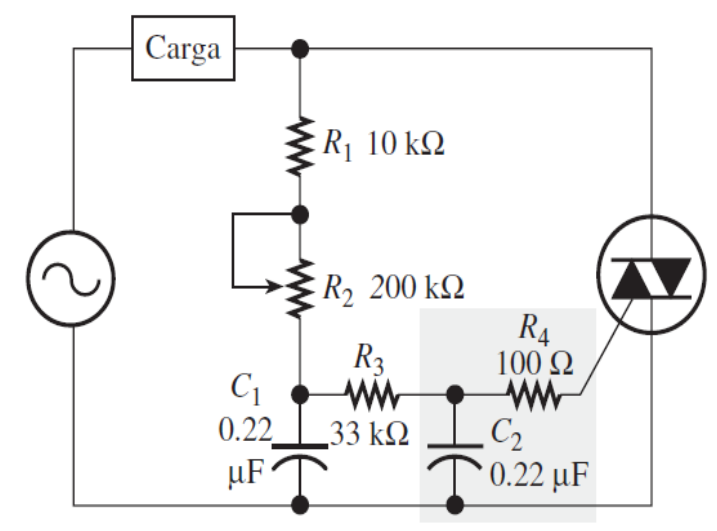

Figura 6 Circuito mejorado de control de compuerta para un TRIAC (Maloney, 2006)

La brusca conexión a red de circuitos próximos con carga inductiva, o las variaciones bruscas de una fuerza contra electromotriz (de una carga constituida, por ejemplo, un rotor de un motor con colector) puede dar lugar a variaciones bruscas de tensión de alimentación del tiristor o TRIAC. El método más corriente empleado para limitar la velocidad de subida de la tensión consiste en colocar un condensador en paralelo con el tristor.

Es pues necesario limitar la corriente de descarga situando un resistor $\mathrm{R}$ (de 20 a 100 ohms) en serie con el condensador. El circuito $\mathrm{RC}$ (red snubber) resultante debe colocarse en paralelo con el tiristor (TRIAC), o lo más cerca posible de él (CENTRO DOCUMENTACIÓN DE ESTUDIOS Y OPOSICIONES, s. f.). En la figura 7 se muestra la red conectada en paralelo con el TRIAC.

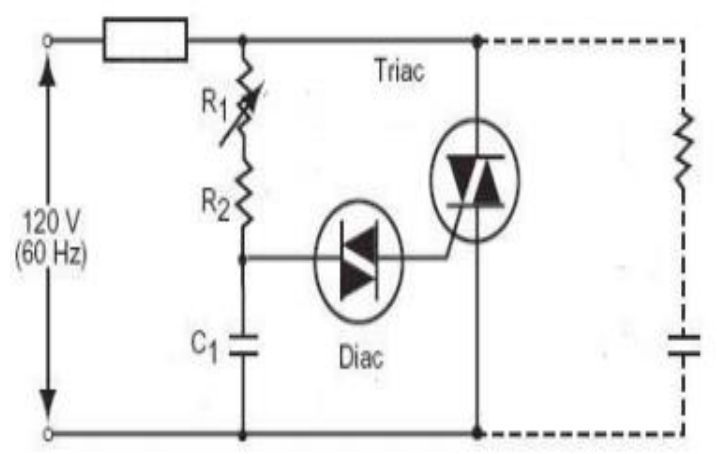

Figura 7 Red de snubber conectada con el TRIAC (Castro et al., 2017)

Como resultado del análisis de los circuitos anteriores se diseñó el circuito que se muestra en la figura 8 debido a que las cargas inductivas tienen el mal hábito de devolver una parte de la energía almacenada en el devanado, de vuelta a los rieles de suministro.

El potenciómetro y el capacitor 2 son utilizados para que el TRIAC sea disparado, ya que éste controla el paso de la corriente alterna a la carga conmutando entre los estados de conducción (activación de carga) y de corte (desactivación de la carga) durante los semiciclos negativos y positivos de la señal de alimentación (110/220 VAC) y la señal de corriente alterna.

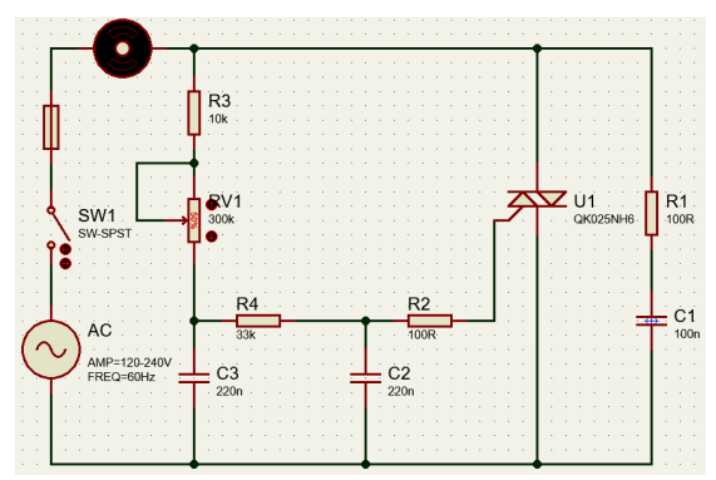

Figura 8 Circuito elegido como arrancador suave

El capacitor en un circuito de corriente alterna tiene su voltaje atrasado con respecto a la señal de entrada.

Cambiando el valor del potenciómetro se modifica la razón de carga del capacitor, el atraso que tiene y por ende el desfase con la señal alterna de entrada. Esto permite que se pueda tener el control sobre la cantidad de corriente que pase a la carga y así la potencia que, en esta, se va a consumir. 
Para el desarrollo del circuito se utilizó el TRIAC BT139-600 que soporta corrientes de 25 Amperes.

\section{Análisis de pruebas realizadas con motores diferentes de baja potencia}

Los datos de las pruebas realizadas fueron medidos con un multímetro digital de la marca STEREN modelo MUL-285 para mediciones de voltaje y corriente; mediante un osciloscopio digital de la marca TEKTRONIX modelo TBS 1052B-EDU se observó el comportamiento del control de fase ajustada mediante el uso del circuito desarrollado, estos instrumentos se utilizaron en las pruebas de arranque directo y de arranque suave.

\section{Prueba en arranque directo}

Al realizar la prueba se identificó que en el arranque del motor se genera un elevado consumo de energía y como se muestra en la figura 9, el arranque directo es de 0.07 Amperes con un voltaje de 126.9 Volts que se muestran en la figura 10.

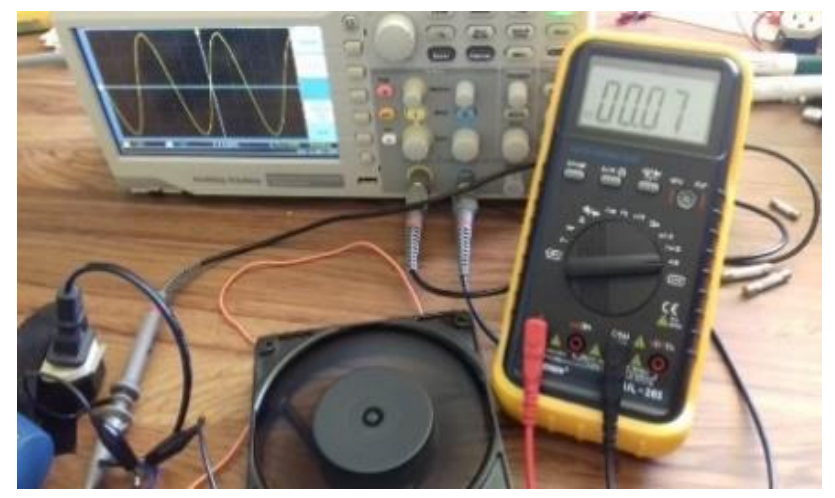

Figura 9 Medición de corriente en arranque directo

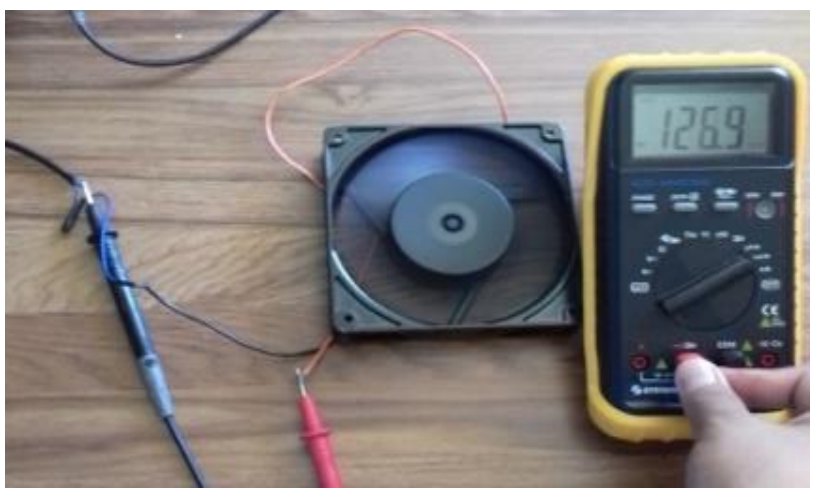

Figura 10 Medición de voltaje en arranque directo

\section{Prueba con arranque Suave}

A continuación, se muestran las mediciones que se realizaron con un multímetro y un osciloscopio digitales en la prueba de arranque suave.

Como se puede observar en las figuras 11 a 18 se muestran las mediciones realizadas a la activación del ventilador que se realiza desde cero y conforme se vaya ajustando la velocidad del motor con el potenciómetro llegara hasta su máxima capacidad.

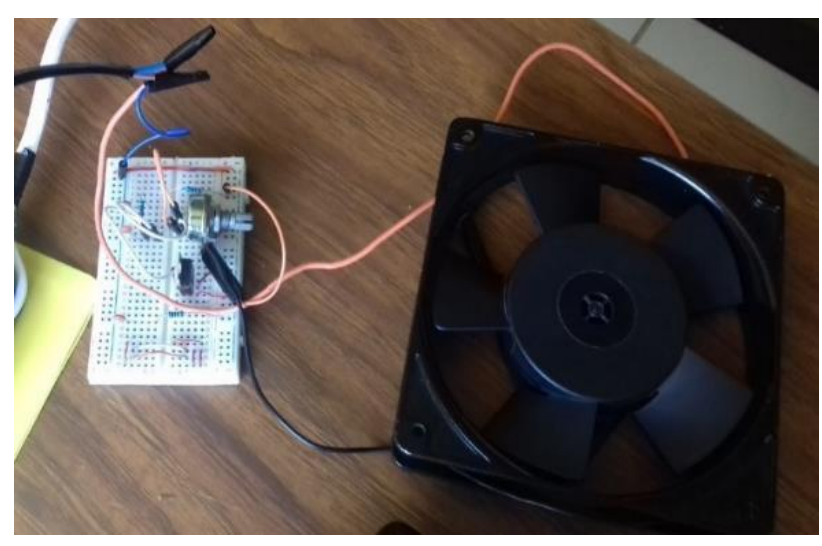

Figura 11 Conexión del motor al arrancador suave

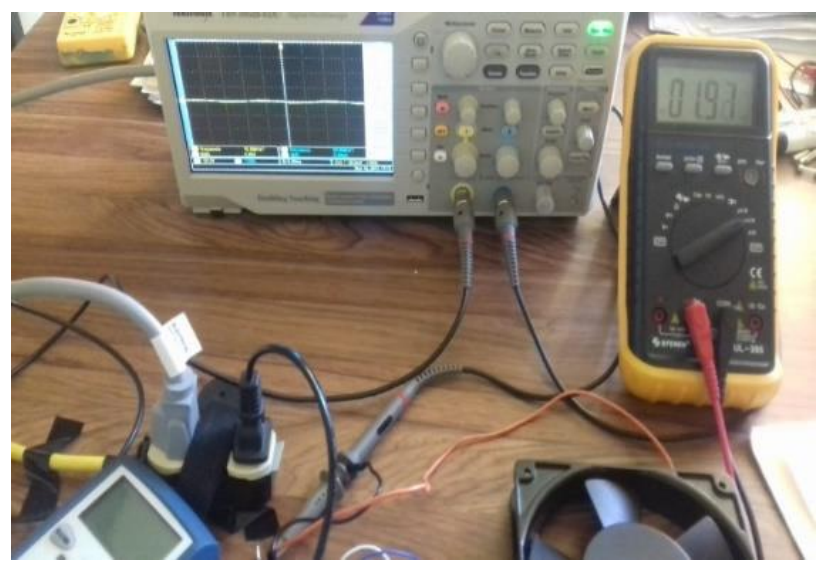

Figura 12 Mediciones al comenzar el arranque del motor. Medición 1

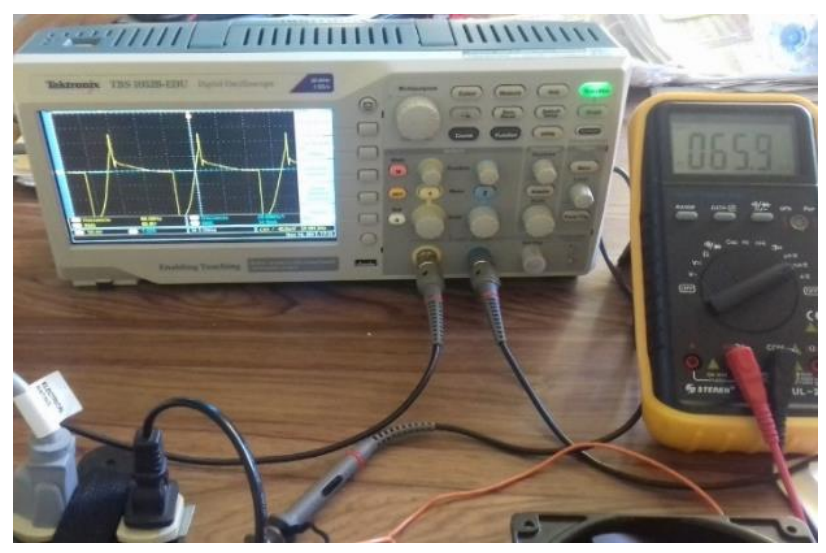

Figura 13 Mediciones con incremento de velocidad en el arranque del motor. Medición 2

BRAVO-TAPIA, Jeremías, GARCÍA-MARTÍNEZ, Eric, RAMOSGUZMÁN, Francisco y ESTÉVEZ-MARTÍNEZ, Yoxkin. Circuito de arranque suave para un motor monofásico de corriente alterna conectado a un Sistema Fotovoltaico. Revista de Innovación Sistemática. 2019. 


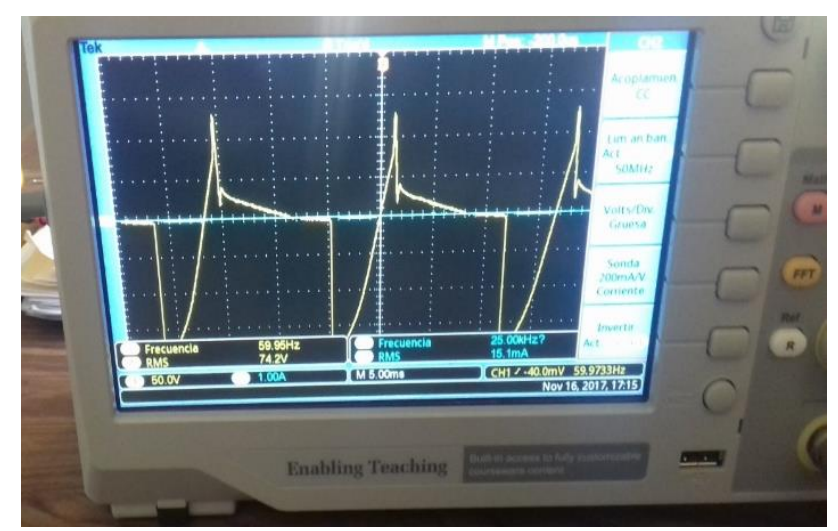

Figura 14 Gráfica de medición al incrementar la velocidad. Medición 2

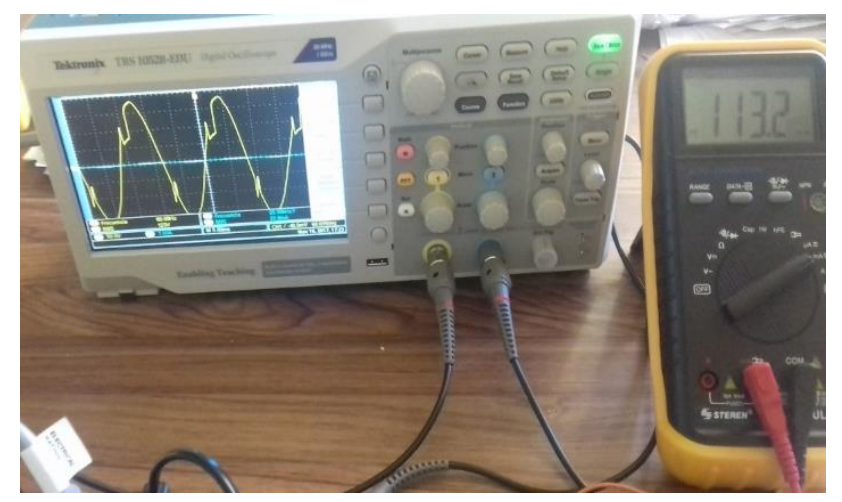

Figura 15 Mediciones con incremento de velocidad en el arranque del motor. Medición 3

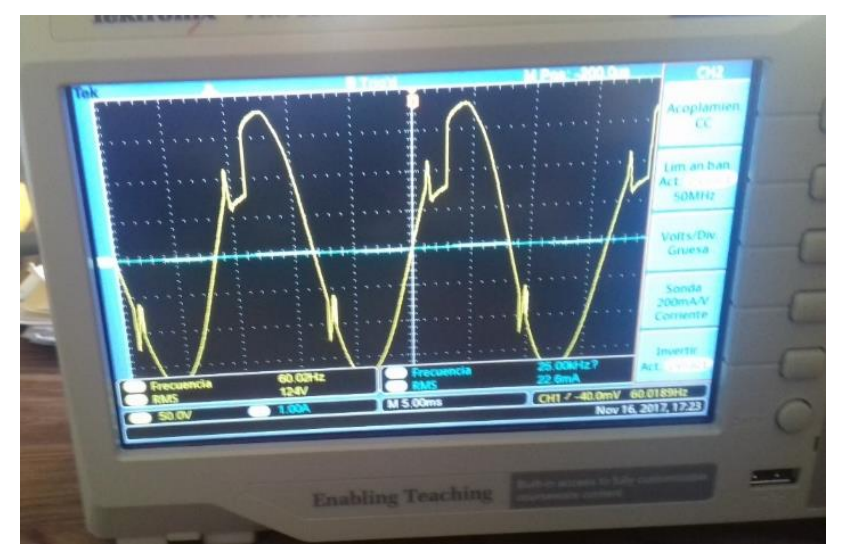

Figura 16 Gráfica de medición al incrementar la velocidad. Medición 3

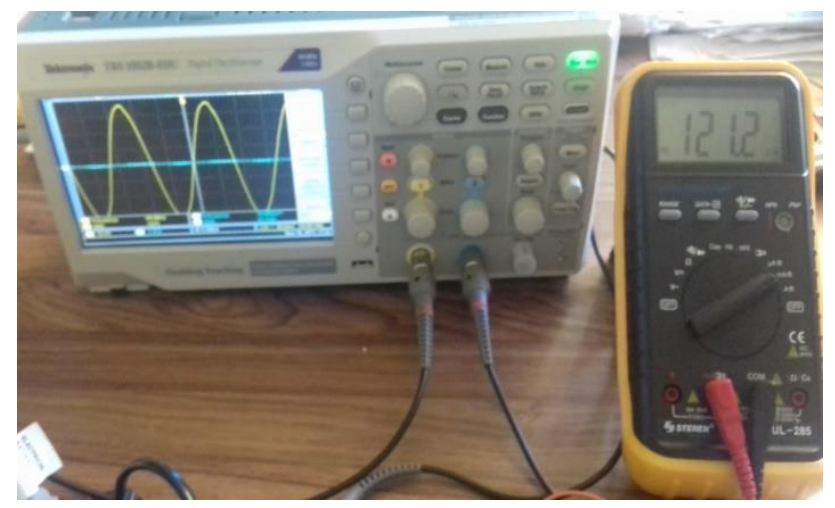

Figura 17 Mediciones con incremento de velocidad al $100 \%$ en el motor. Medición 4

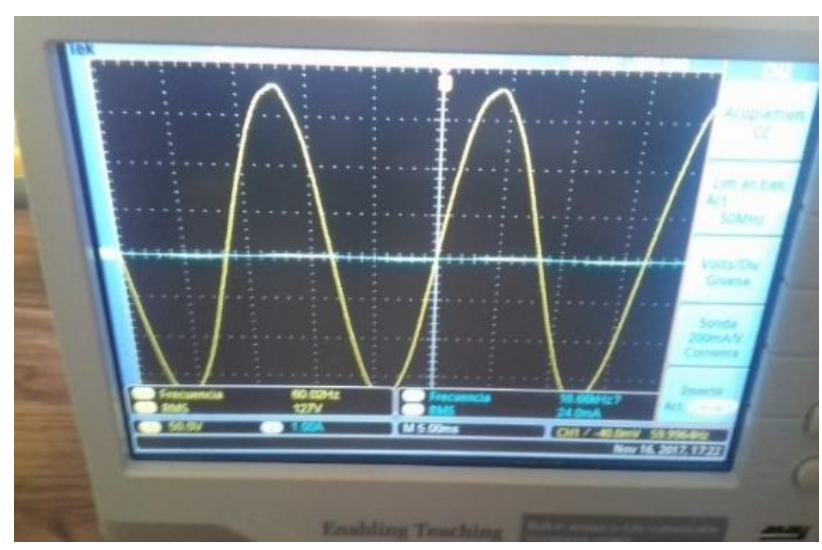

Figura 18 Gráfica de medición al incrementar la velocidad al $100 \%$. Medición 4

Con las pruebas realizadas al visualizar las mediciones y el comportamiento en el control de la fase se logra identificar que con el circuito de arranque suave nos permite manipular la velocidad del motor y al mismo tiempo reduce el consumo de energía que este necesita para alcanzar su máxima velocidad. A continuación, en las figuras 19 a 21 se muestran las pruebas y mediciones realizadas en arranque directo con un motor de $120 \mathrm{~V}$ de CA a $1 / 4 \mathrm{Hp}$. En este caso las mediciones se realizan con un amperímetro digital de potencia de la marca UNI-T, modelo UT231, y se visualizan con el osciloscopio utilizado en las mediciones anteriores.

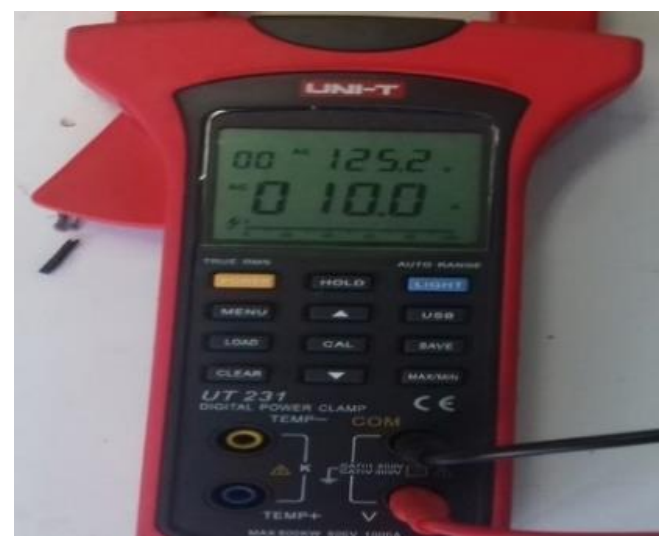

Figura 19 Mediciones de voltaje y corriente en arranque directo

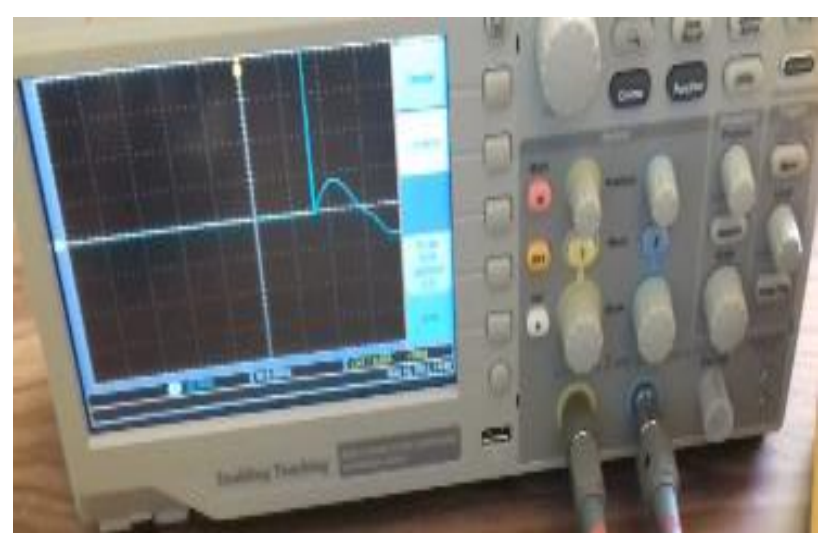

Figura 20 Medición del comportamiento de la energía en arranque directo

BRAVO-TAPIA, Jeremías, GARCÍA-MARTÍNEZ, Eric, RAMOSGUZMÁN, Francisco y ESTÉVEZ-MARTÍNEZ, Yoxkin. Circuito de arranque suave para un motor monofásico de corriente alterna conectado a un Sistema Fotovoltaico. Revista de Innovación Sistemática. 2019. 


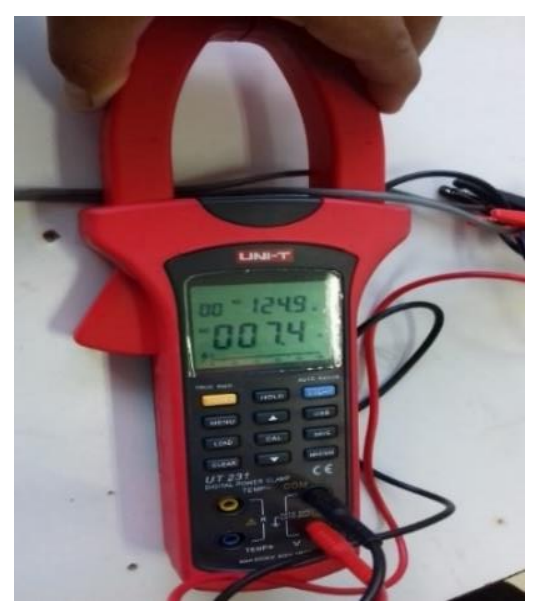

Figura 21 Medición de potencia en arranque directo

Se observa un alto pico de corriente en el arranque del motor, en este caso son hasta $10 \mathrm{~A}$; los niveles de estabilidad del motor no son muy favorables y se mantiene así hasta que se desconecta de la corriente eléctrica. Al conectar el motor con el arranque suave y al inversor se puede observar las diversas variaciones de velocidad que se presentan en el arranque ya que permite controlar la velocidad e incrementar la potencia del motor en un corto lapso de tiempo sin causar daños en el inversor; este comportamiento se observa en las figuras 22 a 26.

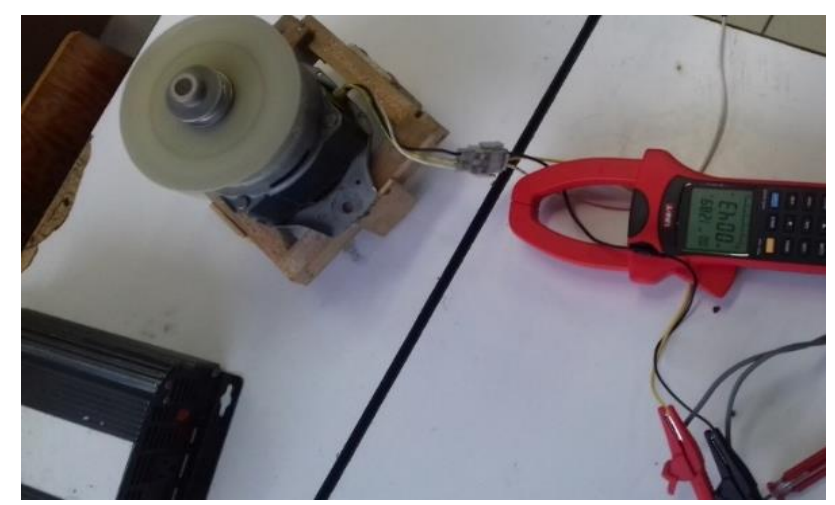

Figura 22 Arranque del motor conectado al Arrancador Suave

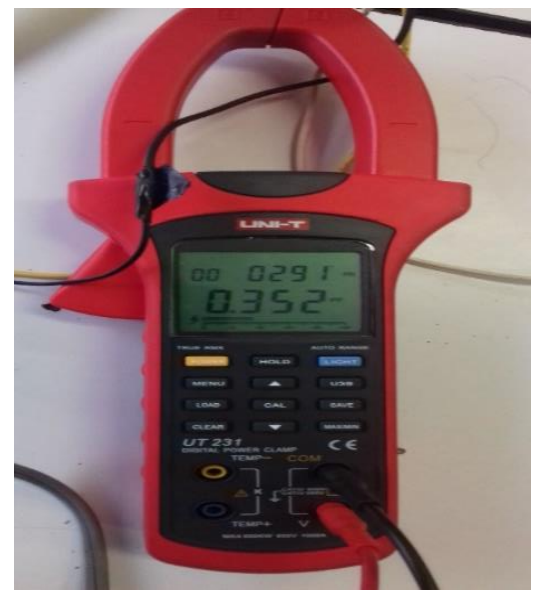

Figura 23 Medición de potencia

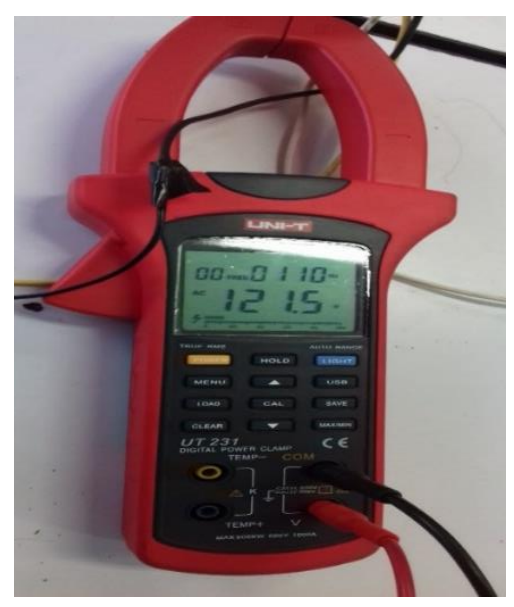

Figura 24 Medición de voltaje

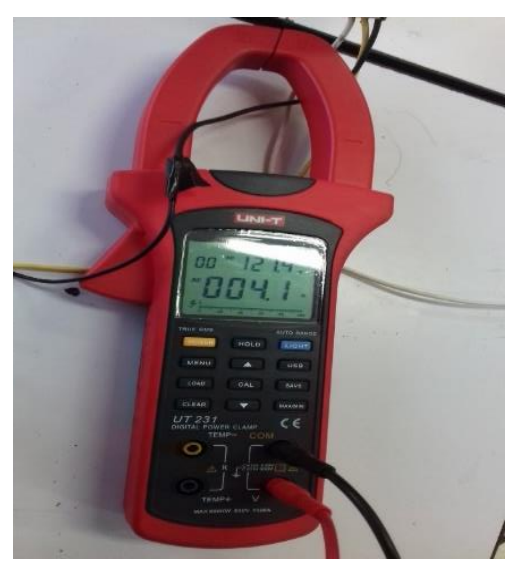

Figura 25 Medición de corriente mínima al estar activo al $100 \%$

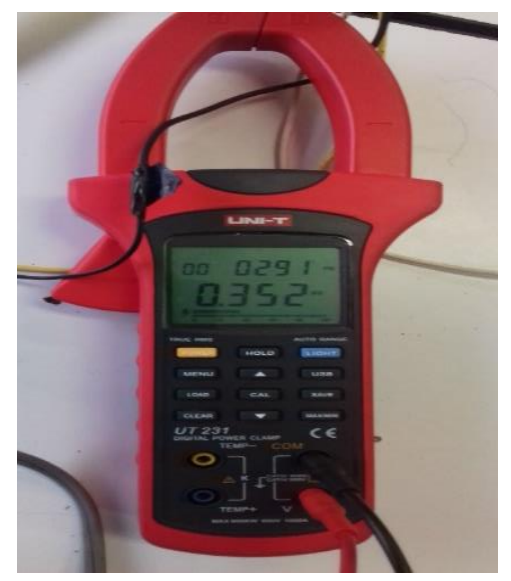

Figura 26 Medición de factor de potencia

\section{Resultados}

El arrancador suave se conectó a un inversor de 1,500 Watts con el cual se logró demostrar que se puede lograr el arranque suave de un motor monofásico. Ésta es una de las primeras pruebas realizadas, aún se está trabajando en la segunda versión con una mejora en el circuito que dará un mejor rendimiento en su funcionamiento mejorando la técnica utilizada. 
Se identificó que la corriente que demanda el motor conectado con el Arranque Suave es más factible que en Arranque Directo; la corriente que demanda el motor cuando se estabiliza es muy baja comparada con el de arranque directo, es decir que el motor puede trabajar a máxima capacidad sin quemarse por el sobrecalentamiento que se genere. La diferencia entre la potencia es mucha ya que la potencia con la cual inicia el arranque suave es de 180 watts que se muestra en la figura 27 y la del arranque directo es de 930 watts como se observa en la figura 28; provocando que el motor se caliente en el arranque y genere un consumo mayor de corriente; en cambio, el arranque suave no genera calentamiento en el motor y conforme la perilla del potenciómetro se regula la velocidad del motor incrementa sin alterar bruscamente la corriente.

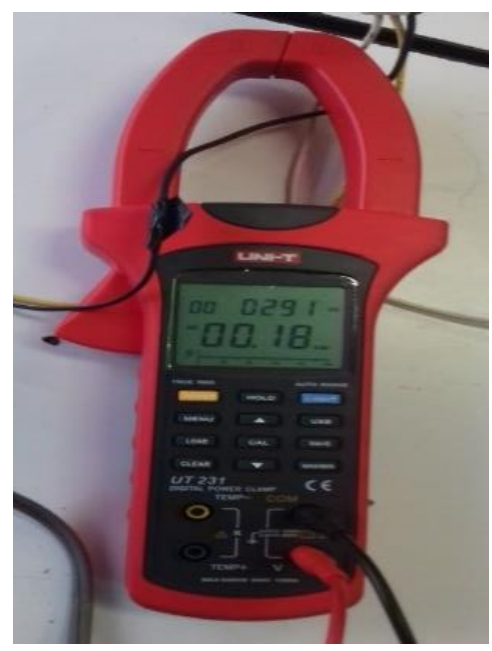

Figura 27 Medición de potencia en arranque suave de 180 W

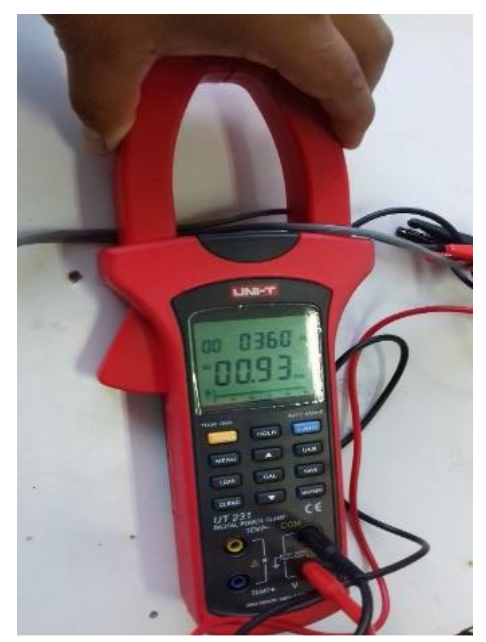

Figura 28 Medición de potencia en arranque directo de $930 \mathrm{~W}$

En la figura 29 se muestra el armado de circuito completo. El cual estará conectado al inversor de corriente DC/AC con un motor de $1 / 4$ Hp.

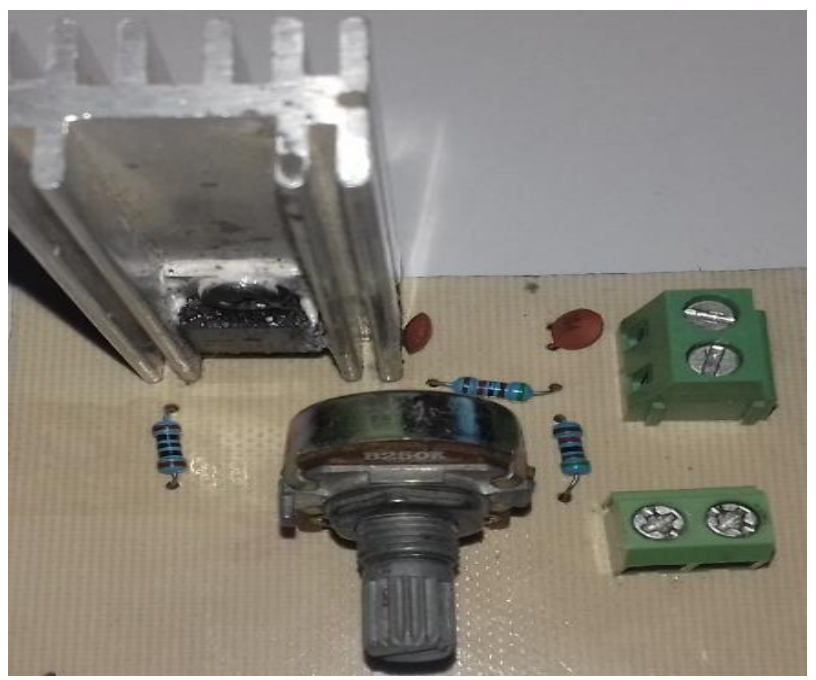

Figura 29 Circuito Arrancador Suave

En la figura 30 se muestra la conexión completa del arrancador suave con el inversor de la marca SAMLEX AMERICA INC modelo SAM-1500-12, del sistema fotovoltaico.

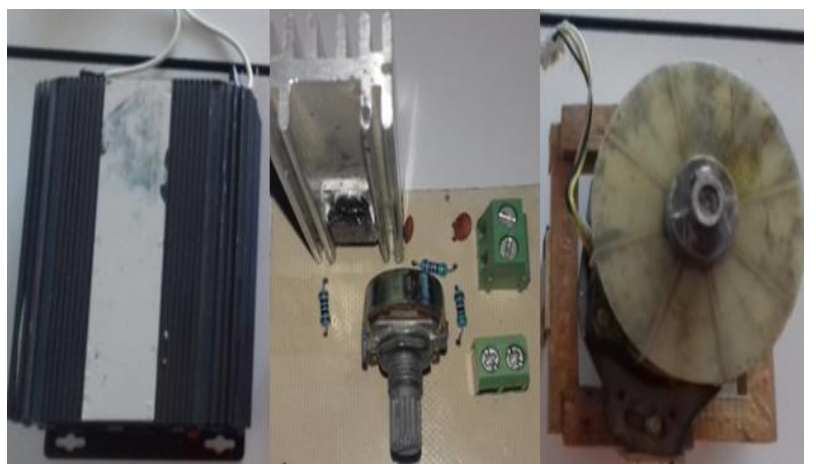

Figura 30 Conexión del Arrancador Suave con el inversor DC/AC

Posteriormente a este resultado se ha realizado una inspección del funcionamiento de un controlador de bombeo fotovoltaico en el que se logró identificar que efectivamente transforma la corriente directa procedente de los paneles a corriente alterna para alimentar una bomba sumergible; se visualizó que cuenta con una red RC para el arranque del motor y protección del circuito con arreglo de relevadores sin TRIAC. En las figuras 31 a 33 se muestran las pruebas realizadas como identificación de componentes y etapas de funcionamiento del controlador en el Laboratorio de electrónica del ITSAO, así como el desmontaje y montaje en el sistema de bombeo fotovoltaico. 


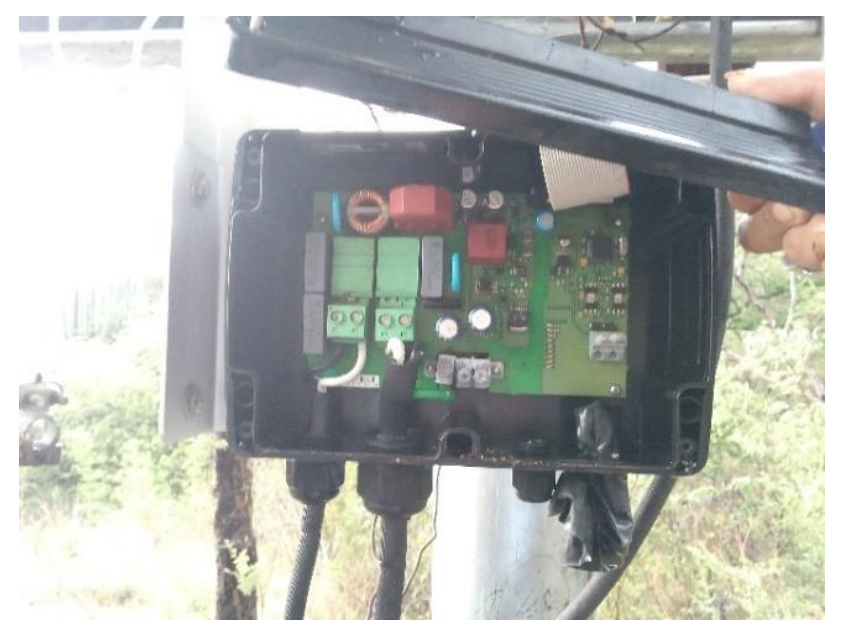

Figura 31 Identificación de los componentes y etapas del controlador

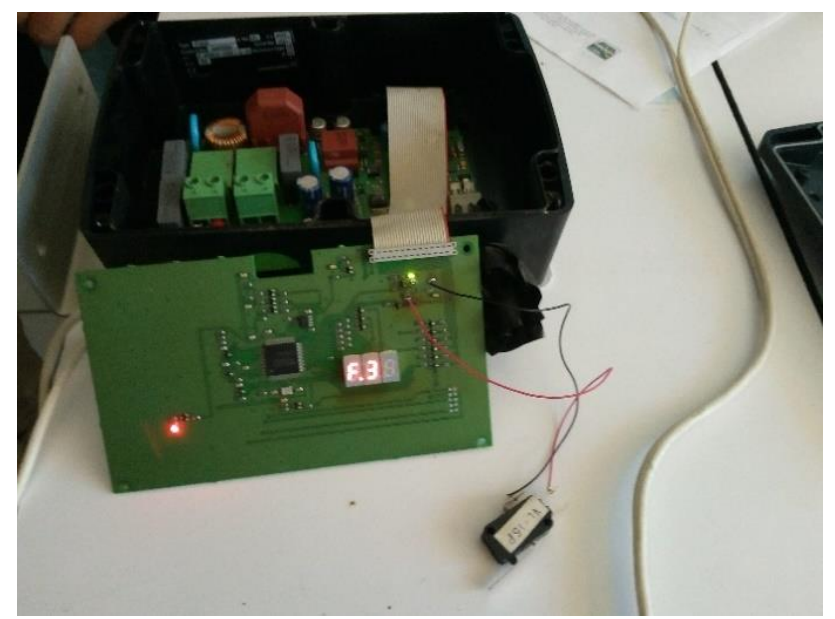

Figura 32 Pruebas realizadas de funcionamiento en el laboratorio de Electrónica del ITSAO

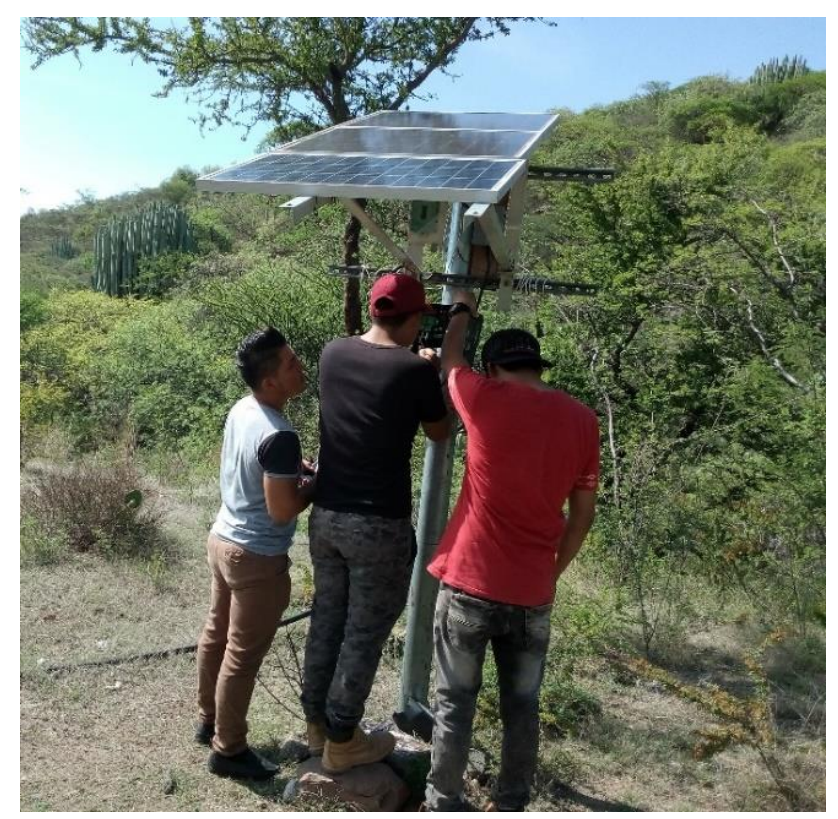

Figura 33 Desmontaje y montaje del controlador al sistema de bombeo fotovoltaico

\section{Conclusiones}

En la actualidad los diversos tipos de arrancadores suaves, sólo son para motores de inducción conectados a fuentes trifásicas, es decir, que sólo son diseñados para este tipo de motores que son usados en la industria. La utilización de un arrancador suave en motores monofásicos es de gran ayuda ya que es capaz de disminuir el consumo de la energía de arranque que estos motores tienen y a su vez se puede incrementar la velocidad del motor desde cero hasta la máxima capacidad sin provocar un alto pico de corriente en el arranque, por lo tanto, permitirá disminuir el costo tan elevado que le genera al consumidor al comprar un inversor de alta potencia.

Con el desarrollo de ésta investigación se han sentado las bases principales para diseñar un circuito de arranque suave para contrarrestar la marcha inicial de un motor monofásico de corriente alterna conectado a un sistema fotovoltaico mediante dispositivos electrónicos; se lograron obtener los resultados teóricos, simulados y prácticos, para que en un futuro, este trabajo sea mejorado implementándose completamente mediante el desarrollo de un controlador completo que incluya la parte de un inversor de onda pura con arrancador suave para motores monofásicos parecido al controlador de los sistemas de bombeo fotovoltaico.

Las corrientes de arranque en los motores monofásicos causan calentamiento provocando daños en el aislamiento de los devanados. Por esta razón se deben instalar elementos que controlen el arranque de los motores.

\section{Sugerencias para el trabajo.}

Se sugiere que para trabajos futuros se mejore el desempeño del circuito incluyendo la incorporación de opto-acopladores, que presentan la ventaja de tener entradas que están eléctricamente aisladas de la corriente alterna o del circuito de potencia realizando una etapa del control mediante la programación de un microcontrolador con ventajas de la capacidad de eliminar el ruido, manejarse a altas frecuencias y posiblemente más precisos al realizar la misma función de arranque de motores cambiando el TRIAC para ajustarlo al tamaño de la carga. 
También se sugiere mejorar el circuito armado en esta primera prueba agregándole un DIAC que presentaría un disparo con un estallido de voltaje corto y consistente en lugar de cambiar bruscamente los pulsos, que a su vez permite que el TRIAC se dispare con transiciones más suaves, causando transitorios y picos mínimos; quedando como un controlador de velocidad para altas cargas inductivas.

En lugar del potenciómetro para el ajuste manual agregarle posiblemente una red de TRIAC's con una configuración de arranque en varios tiempos para permitir un arranque de velocidad gradual por milésimas de segundo hasta alcanzar la velocidad máxima automáticamente.

\section{Referencias}

Adding a Soft Start to Water Pump Motors Reducing Relay Burning Problems | Homemade Circuit Projects. (s. f.). Recuperado de https://www.homemade-circuits.com/addingsoft-start-to-water-pump-motors/

Arrancador suave de estado sólido monofásico Carlo Gavazzi RGTS - Informática industrial Arrancador suave de estado sólido monofásico. (s. f.). Recuperado de https://www.interempresas.net/Informatica_Ind ustrial/FeriaVirtual/Producto-Arrancadorsuave-de-estado-solido-monofasico-CarloGavazzi-RGTS-173442.html

Ayyildiz, M., Shok, F., Soygenc, O. C., \& Ergene, L. T. (2018). Soft Starter Circuit Design for Single Phase Squirrel Cage Induction Motor. 2018 20th International Symposium on Electrical Apparatus and Technologies (SIELA), 1-4. doi:10.1109/SIELA.2018.8447074

Bhagyashree, S. M. M., \& Khule, S. S. (2017). Design of solar water pumping system with FCMA soft starter. 2017 International Conference on Computing Methodologies and Communication (ICCMC), 951-954. doi: 10.1109/ICCMC.2017.8282608

Boylestad, R. L., \& Nashelsky, L. (2009). Electrónica: Teoría de circuitos y dispositivos electrónicos (10 ed.). México: Pearson Educación.
Castro, J. D. Á., Rios, O. A. de los, \& Merino, Y. (2017). Dispositivo electrónico para controlar la frecuencia en un motor monofásico de corriente alterna. Scientia Et Technica, 22(4), 308-314.

CENTRO DOCUMENTACIÓN DE ESTUDIOS Y OPOSICIONES. (s. f.). Tema 11. Análisis de circuitos electrónicos para el control de potencia. Dispositivos y elementos utilizados. Clasificación, función, tipología y características. Criterios y procedimientos utilizados para el diseño de estos circuitos. Recuperado de https://www.serina.es/empresas/cede_muestra/2 12/TEMA\%20MUESTRA.pdf

EasyStart 364 (3-ton) Single Phase Soft Starter for Air Conditioners - Micro-Air, Inc. (s. f.). de https://www.microair.net/products/easystart364-3-ton-single-phase-soft-starter-for-air conditioners? variant $=30176048267$

González Ponce Abraham. (2016). Bombeo Solar con Variadores-de Frecuencia para Aplicaciones de Regadio (Tesis). Universidad Rey Juan Carlos.

Khan, R. A., Khan, L. A., \& Hussain, S. Z. (2015). Design and Simulation of $0.75 \mathrm{hp} \mathrm{Soft}$ Start AC Water Pump Powered by PV Solar System. Universal Journal of Mechanical Engineering, 3(4), 113-121. doi: 10.13189/ujme.2015.030401

Kling, S. (2002). Nuevos arrancadores suaves para motores eléctricos. $A B B$ Control $A B, 1,56$ 63.

Ludeña Jimenez, M. E., \& Lapo Camisan, D. L. (2018). Tesis. Recuperado a partir de http://dspace.ucuenca.edu.ec/handle/123456789 /31324

Maloney, T. J. (2006). Electrónica Industrial Moderna (5ta ed.). México: Pearson Educación. Miguel Alonso, A., \& Faustino Chenlo, R. (s. f.). Sistemas de Bombeo Fotovoltaico. Recuperado de

https://www.eoi.es/es/file/18718/download?tok en $=36 \mathrm{ZyZ} 5 \mathrm{Nj}$ 
Miguel Llopis Morales. (2018). Diseño de una instalación solar fotovoltaica para bombeo de $55 \mathrm{~kW}$ para el riego de la Huerta de Alicante en Mutxamel. (Tesis de maestría, Escuela Técnica Superior Ingenieros Industriales de Valencia). Recuperado

https://riunet.upv.es/handle/10251/110369

Neri, A. L., \& Lyra, A. C. C. (2006). Starting Control Using Angle Adjustment of the Double Dimmer Supply Method for Single-Phase Induction Motor. 2006 12th International Power Electronics and Motion Control Conference, 979-983.

doi:10.1109/EPEPEMC.2006.4778527

Orellana Zea, A. A., \& Pintado Garate, I. E. (2019). Desarrollo de un microinversor para la optimización del arranque en motores monofásicos. Recuperado de http://dspace.ups.edu.ec/handle/123456789/170 08

$\mathrm{T}$

ODOPRODUCTIVIDAD: La tecnología del arranque suave de los motores. (2011, marzo 12). Recuperado de TODOPRODUCTIVIDAD website:

http://todoproductividad.blogspot.com/2011/03/ la-tecnologia-del-arranque-suave-de-los.html 